(NASA-TM-111139) AN OVERVIEW OF

N96-14464 ENGINEERING CONCEPTS AND CURRENT OESIGN ALGORITHMS FOR PROBABILISTIC STRUCTURAL ANALYSIS INASA. LEWIS Research (enter) $14 \mathrm{p}$

Unclas

$63 / 390077068$ 



\title{
AN OVERVIEW OF ENGINEERING CONCEPTS AND CURRENT DESIGN ALGORITHMS FOR PROBABILISTIC STRUCTURAL ANALYSIS
}

\author{
S.F. Duffy ${ }^{1}$, J.Hu ${ }^{2}$ \\ Civil Engineering Department \\ Cleveland State University \\ Cleveland, Ohio \\ D.A. Hopkins ${ }^{3}$ \\ Structural Mechanics Branch \\ NASA Lewis Research Center \\ Cleveland, Obio
}

\begin{abstract}
The article begins by examining the fundamentals of traditional deteministic design philosophy. The initial section outlines the concepts of failure criteria and limit state functions, two traditional notions that are embedded in deterministic design philosophy. This is followed by a discussion regarding safety factors (a possible limit state function) and the common utilization of statistical concepts in deterministic engineering design approaches. Next, the fundamental aspects of a probabilistic failure analysis are explored, and it is shown that deterministic design concepts mentioned in the initial portion of the article are embedded in probabilistic design methods. For components fabricated from ceramic materials (and other similarly brittle materials) the probabilistic design approach yields the widely used Weibull analysis atter suitable assumptions are incorporated. The authors point out that Weibull analysis provides the rare instance where closed form solutions are available for a probabilistic failure analysis. Since numerical methods are usually required to evaluate component reliabilities, a section on Monte Carlo methods is included to introduce the concept. The article concludes with a presentation of the technical aspects that support the numerical method known as fast probability integration (FPI). This includes a discussion of the Hasofer-Lind and RackwitzFiessler approximations.
\end{abstract}

\section{INTRODUCTION}

Most parameters that are incorporated into engincering analyses have to a greater, or lesser extent, some level of uncertainty. In order to achieve a general accounting of the entire spectum of values that design parameters exhibit (especially for those cases where one or more of the design parameters exhibits substantial scatter) a design engineer should utilize probabilistic methods. However, a reliability approsech to engineering design demands that an engineer must tolerate a finite risk of unacceptable performance. This risk of unacceptable performance is identified as a component's probability of fallure. The primary concem of the engineer is minimizing this risk in an economical manner. To accomplish this requires analytical tools that quantify uncertainty in a rational fashion. The tools for dealing with uncertainty in a rational fashion have been developed in a field of mathematics known as probability theory. Since entire texts are dedicated to this field, only those concepts that are applicable to the design of engineered components are presented here.

In order to meet the numerical needs that accompany a probabilistic analysis several research teams sponsored by NASA Lewis Research Center (LeRC) have focused on the development and application of reliability design algorithms. Two of these groups have produced program deliverables that include reliability models and computer software. Specifically, one program focuses on the engineering analysis of components fabricated from ceramic materials. A number of reliability models developed for ceramic materials (sce Duffy et al., 1992, for an overview) have been incorporated into public domain computer algorithms such as the CARES (Ceramics Analysis and Reliability Evaluation of Structures), T/CARES (Toughened Ceramics Aralysis and Reliability Evaluation of Structures) and C/CARES (Composite Ceramics Analysis and Relinbility Evaluation of Structures). These computer algorithms are coupled to an

'Associate Professor, ${ }^{2}$ Graduate Assistant, 'Acting Branch Chief 
assortment of commercially available general purpose finite element programs. The algorithms yjeld quasi-static component reliability. In sddition the CARES family of software includes parameter estimation modules that allow the design engineer to evaluate the strength distribution parameters from failure dats. It is assumed that for this type of reliability analysis the failure strength of the material can be characterized by either a two- or three-parameter Weibull distribution.

The second program that has developed probabilistic tools for design engineers is the Probabilistic Structural Anslysis Methods (PSAM) program. This endeavor takes on a more global perspective and deals with the stochastic nature of design parameters in a general fashion. In a manner similar to the structural ceramics effort mentioned above, this program integrates probabilistic algorithms with structural analysis methods. The primary result is the NESSUS (Numerical Evaluation of Stochastic Structures Under Stress) computer software.

This article presents the underlying engineering concepts that support the technical aspects of both the CARES and PSAM programs. In addition, the authors outline the commonality between the programs by demonstrating the shared technical principles. Specific details regarding the CARES family of software algorithms and the NESSUS software are incorporated into the conference presentation that accompanies this article.

\section{FAILURE CRITERION \& LIMIT STATE FUNCTION}

The suceess of a structural analysis hinges on the appropriate choice of design variables used to describe the overall thermo-mechanical behavior of a component. The design variables can include, but are not limited to, strength parameters, extemal loads, allowable deformations at predetermined locations in the component, cycles-to-failure, and material stifiness properties. After the engineer has determined what design variables are pertinent to a given class of design problems, they can be assembled in an $N$-dimensional vector. This vector of design parameters can be identified as

$$
y_{.}=\left(y_{1}, y_{3}, \ldots y_{N}\right)
$$

Design variables can easily interact with one another, thus a functional relationship is needed to describe any interaction. This function is most commonly referred to as a failure criterion. Common examples include strength based criterion such as the maximum distortional energy criterion and the Mohr-Coulomb criterion; fatigue failure criterion are represented by Miner's rule; and fracture criterion include the critical strain energy release rate method and the stress intensity factor methods. Usually a failure criterion represents the first step in defining a limit state. If an operational state for a structural component falls within the boundaries of a limit state, the performance of the strustural component is acceptable. An operational state for a component that falls on the boundary of a limit state denotes fallure. For the failure eriterion just cited delineztion between acceptable performance and failure is made at a point in the component.

A fallure criterion and limit state function can be expressed by the general formulation

$$
8=8\left(y_{n}\right)
$$

Note that $\boldsymbol{g}$ defines a surface in an $N$-dimensional design variable space. Once again this function must stipulate how each design variable interacts in producing failure. Here values of $g>0$ indicate a safe structure, whereas values of $g \leq 0$ correspond to a failed structure. The failure criterion (or a limit state function) can be defined by either a complete loss of load carrying capacity, or alternatively by a loss in serviceability. The conceptual distinction between a limit state function and a failure criterion is based on scale. It was indieated above that 2 failure criterion focuses on a point. Limit state functions focus on the component or structure. Often times there is no difference between the two since failure at a point constitutes failure of the component. In contrast, consider a structural component where plastic yield is a possible failure mode. If yielding (failure) at a point is described by a yield function, then this function represents the failure criterion for this particular mode of failure. Yet a structural component may not fail if yielding has occurred only at a point. In fact the component may continue to function safely until a sufficient number of plastic hinges have formed and the structure collapses. The formation of a sufficient number of plastic hinges is described mathematically by a limit state function. The reader is directed to the extensive literature that followed Drucker's initial work (1952) in establishing bounds on linit state functions for this type of failure analysis. However, the point is that for a plasticity analysis the failure criterion (i.e., the yield criterion) is different from the linit state function.

As a prelude to the discussion that follows later conceming Weibull analysis, a structural component fabricated from a ceramic material is treated as a weakest-link system. If one link in the chain fails, the entire chain fails. This assumption gives rise to a particular modeling approach in ealculating component reliability. It also infers that failure at a point constitutes component failure. In this sense the failure criterion and the limit state function will be one in the same.

\section{SAFETY FACTORS \& DETERMINISTIC FAILURE} ANALYSIS

To begin contrasting the difference between deterministic and probabilistic failure analyses the discussion in this section is focused on a specific failure mode, i.e., the exhaustion of strength capacity. A structural component can thil when it encounters an extreme load, or when a combination of loads reaches a critical collective magnitude, and the ability to withstand the applied load is exhausted. With the design algorithms presented in this article the engineer ean easily 
quantify the msgnitude of the extreme load event leading to Filure, and account for the frequency at which this extreme event occurs. In addition, the strength (or capacity) of the material and any variation in this design parameter can be quantified. A brief discussion regarding safety factors (as well as safety margins) follows. This discussion underscores the need to account for variability of design parameters in a coherent manner. These quantities are typically utilized in deterministic designs, and are essily incorporated into probabilistic designs.

In the field of structural mechanics it is customary to define safety factors (and sometimes safety margins) in order to ascertain how "close" a component is to falling. If $L$ represents the load on a component, and $R$ represents the resistance (or capacity) of the material, then the safety factor is defined as

$$
\text { S. F. }=\frac{R}{L}
$$

Alternatively, the safery margin can be utilized, and this measure is defined as

$$
\text { S. } M .=R-\boldsymbol{Z}
$$

Failure occurs when the safery factor falls below one, or when the safety margin falls below zero. These two expressions represent the simplest and most fundamental definition of 2 limit state. In the following section where the principles of probabilistic failure analysis are outlined, these two expressions are ubilized to explain basic concepts. However, to employ either safety factors or safety margins the design engineer must quantify parameters $R$ and $L$. Dats must be collected and a single "mostlikely" value must be assigned for each parameter. If the typical structural engineer has been exposed to statistical methods, these concepts were encountered in quantifying material properties from experimental data. Thus the design engineer is familiar with the concept of a central location parameter for experimental data defined by the sample mean, i.e.,

$$
\bar{x}=\left(\frac{1}{N}\right) \sum_{i=1}^{N} x_{1}
$$

A second parameter, the sample variance, serves as a measure of data dispersion. It is defined as

$$
S^{2}=\left(\frac{1}{N-1}\right) \sum_{i=1}^{N}\left[x_{1}-\bar{x}\right]^{2}
$$

In the expressions for the sample mean and variance, $x_{1}$ represents the $i^{\text {th }}$ observation in an experiment with $N$ observations. Other sample descriptors exist (e.g., skewness and kurtosia); however, the mean and the variance are more widely recognized and understood. Usually the gample mean is used to identify a single "rnost-likely" value for a design parameter. The variance is most often utilized in simple minded fashion to indicate how well the experiment is being performed, i.e., small variance indicates good experimental technique. However, this attitude tends to minimize the fact that some design parameters inherently behave in a random fashion. Variation in experimental data can easily be a fundamental property of a particular design parameter, not a commentary on experimental technique.

Often the engineer is not required to determine values for design parameters directly from experiments. Values for resistance parameters cas be obtained from handbooks or existing corporate data bases. Either source of information may concurrently list values for the standard deviation (defined as the square root of the sample variance) but this information is too often ignored in a deterministic failure analysis where the sample mean is used to represent the "most-likely" value of the design parameter. Increasing the mean value of the load parameter by a multiple (usually three) of standard deviations, and decreasing the mean value of resistance parameter by the same multiple of standard deviations is one way of including information regarding data dispersion in a safery factor design. This method, referred to as the three-sigma approach, yields the following definition for the factor of safety

$$
\text { S. } F=\frac{\bar{x}_{R}-3\left(S_{R}\right)^{1 / 2}}{\bar{x}_{L}+3\left(S_{L}\right)^{1 / 2}}
$$

Clearly this definition of the safety factor admits information concerning the data dispersion for both the load and resistance parameters.

However, if either of the design parameters exhibits 2 significant scatter, as evidenced by a relatively large sample standard deviation, then the design engineer must compensate in some manner to maintain a prescribed safecy factor for a component. Thus, in an effort to maintain a given level for the safety factor, analyses predicated on equation ( 7 ) may casily lead to uneconomical designs. Utilizing probabilistic methods can readily compensate for parameter variation, enabling an engineer to further pursue a design that would be otherwise rejected based on traditional methods. In addition, for materials that exhibit size effects where the average strength decreases with specinen size (e.g., ceramic materials) the three-sigma approach leads to a fundamental problem in identifying what value to use for the resistance random variable.

\section{FUNDAMENTALS OF PROBABILISTIC FAILURE ANALYSIS}

Utilization of equation (7) represents an attempt to include more information regarding the true characteristics of 
design prsameters. Momentarily locusing on the resistance parameter, equation (7) implies that the resistance design parameter is inherently multi-valued. If this multi-valued resistance parameter assumes different values at random during strength-to-failure experiments, then the parameter should be treated as random variable. This holds for the load design parameter as well. Specifically note that if the load and resistance design parameters are treated as random variables, then equation ( 7 ) does not include any information on the underlying distribution (c.g., normal, log-normal, Weibull, exponential, etc.) that characterizes the design parameter. As the discussion unfolds in this section the reader will see that reliability methods attempt to overcome this inadequacy.

Consider a component fabricated from a material with a resistance described by the random variable $\boldsymbol{R}$. A single load, represented by the random variable $L$, is applied to the component. Both randorn variables are represented mathematically by distinctly different probability deasity functions (e.g., normal, log-normal, exponential, Weibull, Rayleigh, ete.). The load and resistance random variables can be described by the same type of probability density function as long as the distribution parameters are different.

However, in this discussion the distributions for the random variables are left unspecified. This is intentional in order to simplify and emphasize several issues. Making use of the concept of a safety factor, the probability of failure for a component where a single load is applied is given by the expression

$$
P_{f}=\text { Probabitity }(R / L \leq 1)
$$

Alternatively, the probability of failure can be defined using the safety margin. Here

$$
P_{\rho}=\text { Probability }(R-L \leq 0)
$$

For either definition, $P_{f}$ is the product of two tinite probabilities summed over all possible outcomes. Each probability is associated with an event and a random variable. The first event is defined by the random variable $L$ taking on value in the range

$$
\left(x-\frac{d x}{2}\right) \leq I \leq\left(x+\frac{d x}{2}\right)
$$

The probability associated with this event is the area under the probability density function for the load random variable over this interval, i.e.,

$$
P_{1}=f_{2}(x) d x
$$

The second event is associated with the probability that the random variable $R$ is less than or equal to $x$. This is the area under the probability density function for the resistance random variable over the range from minus infinity (or an appropriate lower limit defined by the range of the resistance random variable) to $x$. This second probability is given by the cumulative distribution function evaluated at $x$, i.e.,

$$
P_{2}=F_{R}(x)
$$

With the probability of failure defined as the product of these two probabilities, summed over all possible values of $x$, then

$$
P_{f}=\int_{-} F_{l}(x) f_{L}(x) d x
$$

To interpret this integral expression, consider Figure 1. This figure contains a graph of an arbitrary probability density function $\left(f_{R}\right)$ for the resistance random variable superimposed on the graph of an arbitrary probability density function $\left(f_{l}\right)$ for the load random variable. Note that $R$ and $L$ must have the same dimensions to plot these two quantities on the same graph. A common misconception is that $P_{f}$ is the area of overlap encompassed by the two probability density functions. Scrutiny of equation (13) leads to the appropriate conclusion that the probability of failure is the area under the composite function

$$
\eta_{R L}(x)=F_{R}(x) f_{L}(x)
$$

Due to the complexities introduced by specifying $F_{R}(x)$ and $f_{L}(x)$ a closed form solution rarely exists for equation (13). One exception is the application of equation (13) to ceramic materials, which is discussed in the next section.

\section{CERAMIC MATERIALS AND SYSTEM RELIABILITY}

Even though variations in loads and strength ean be readily accommodated by the coneepts presented in the previous section, for components fabricated from ceramic materials it is the variation in material strength that dominates the desiga. Lack of ductility combined with flaws, defects, or inclusions that have various sizes and orientations leads to scatter in failure strength. Thus the strength associated with these ceramic materials reflects an intrinsic fracture toughness and a homogeneous distribution of flaws present in the materials. The analytical concepts presented in this section will accommodate this singular focus on strength variation.

Experimental data indicates that the continuous random 


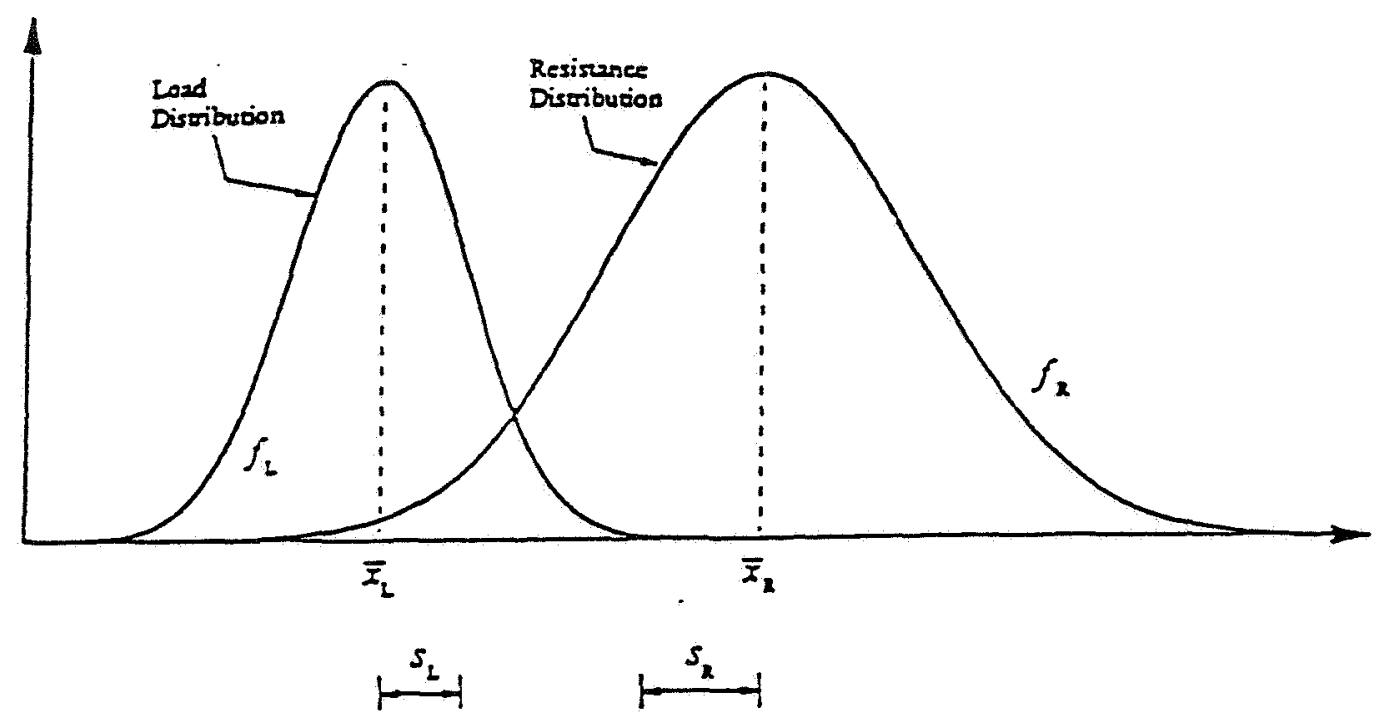

Figure 1 An abitrary load - resistance interference graph

variable representing uniaxial tensile strength (a resistance design parameter) of monolithic ceramics is asymmetrical about the mean and will assume only positive values. These characteristics rule out the use of the nomal distribution (as well as others) and point to the use of the Weibull distribution or a similarly skewed distribution. The three-parameter Weibull probability density function for a continuous random strength variable, denoted as $\mathbf{Z}$, is given by the expression

$$
f_{L}(\sigma)=\left[\frac{\alpha}{\beta}\right)\left[\frac{\sigma-\gamma}{\beta}\right]^{(-1)} \exp \left(-\left(\frac{\sigma-\gamma}{\beta}\right)\right]
$$

for $\sigma>\boldsymbol{\gamma}$, and

$$
f_{z}(\sigma)=0
$$

for $0 \leq \gamma$. In equation (15) $\alpha$ is the Weibull modulus (or the shape parameter), $\beta$ is the Weibull seale parameter, and $\gamma$ is a threahold parameter. If the value of the random variable is below the threshold parameter, the probability density function is zero. Often the value of the threshold parameter is taken to be zero. In component design this represents a conservative assumption, and yields the more widely used two-parameter Weibull formulation.
If the resistance design parameter is characterized by the Weibull distribution and the load design parameter is assumed deteministic, then the following probability density function

$$
f_{2}(x)=8\left(x-x_{0}\right)
$$

is utilized in equation (13) for the load random yariable. Here $\delta$ is the Dirac delta function defined as

$$
8\left(x-x_{0}\right)= \begin{cases}- & x=x_{0} \\ 0 & x=x_{0}\end{cases}
$$

Note that the Dirac delta function satisfies the classical definition of a probability density functions. This function represents the scenario where the standard deviation of a distribution approaches zero in the limit, and the random variable takes on a central value (identified here as $z_{\text {. }}$ ). Insertion of equation (17) into equation (13) yields the following expression for the probability of failure:

$$
P_{f}=\bar{\int}_{-} F_{d}(x) \delta\left(x-x_{0}\right) d x
$$

However, with the Dirac delta function embedded in the integral 
expression, the probability of failure simplifies to

$$
P_{f}=F_{R}\left(x_{0}\right)
$$

Thus the probability of failure is govemed by the cumulative distribution function that characterizes the resistance random variable. This expression (with modification) is fundamental concept associated with Weibull analysis.

Equation (20) yields the probability of failure (after an appropriate distribution has been specified for the random variable $R$ ) for a simple component with a single deterministic load which is identified as $x_{0}$. However, a unique property of ceramic materials is an apparent decreasing trend in strength with an increase in the size of the component. This is the so ealled size effect. As an example, consider that the simple component represents a uniaxial tensile specimen. Now suppose that two groups of these simple componenes exist. Each group is identical with the exception that the size of the specimens in the first group is uniformly smaller than the specimens in the second group. For ceramic materials the sample mean from the first group would be consistently and distinctly larger in a manner that can not be accounted for by randomness. Thus equation (20) must be transformed in some fashion to admit a size dependence. This is accomplished through the use of system reliability concepts. It should be understood that the expression given in equation (20) represents the probability of failure for a uniform set of boundary conditions. If the boundary conditions are modified in any fashion, or the geometry of the component changes, equation (20) is no longer valid. To account for size effects and deal with the probability of failure for a component in a general manner, the component should be treated as a system, and the focus must be directed on the probability of failure of the system.

Typically, for a structural component with a varying stress field, the component is discretized, and the stress field is characterized using finite element methods. Since component failure may initiate in any of the discrete elements, it is easy to consider the discretized component from a systems viewpoint. A discretized component is a series system if it fails when one of the discrete elements fail. This concept gives rise to weakest-link reliability theories. A discretized component is a parallel system when failure of a single element does not necessarily cause the component to fail, since the remaining elements may sustain the load through redistribution. Parallel systems lead to what has been referred to in the literature as "bundle theories." These two types of systems represent the extremes of failure behavior and suggest more complex systems such as " $r$ out of $n$ " systems. Here a component (system) of $n$ elements functions if at least $r$ elements have not failed. However, the failure behavior of monolithic ceramic materials is brittle and catastrophic. This type of behavior fits within the description of a series system, thus ceramic materials are modeled as a weakest-link reliability system.

Now the focus is directed to the probability of failure of a discrete element and how this failure relates to the overall probability of failure of the component. If the fuilure of an individual element is considered statistical event, and if these events are independent, then the probability of tailure of $a$ discretized component that acts as series system is given by

$$
P_{f}=1-\prod_{i}^{N}\left(t-P_{1}\right)
$$

where $N$ is the number of diserete finite elements for a given component. Here the probability of failuse of the $i$ diserete element $\left(\boldsymbol{P}_{1}\right)$ is given by the expression

$$
\mathcal{P}_{1}=\Delta \boldsymbol{V}_{1}
$$

where $\boldsymbol{V}$ denotes volume and is a failure function per unit volume of material. This introduces the requisite size scaling that is associated with ceramic materials. Adopting an argument used by Weibull (1939) where the norm of the $\Delta V_{l}$ 's tends to zero in the limit as $N$ goes to infinity, then the component probability of failure is given by the following expression

$$
P_{f}=1-\operatorname{ex}\left(-\int_{V} \downarrow d V\right)
$$

What remains is the specification of the failure function $\downarrow$. The most basic formulation for $\psi$ is given by the principle of independent action (PLA). For this reliability model

$$
\downarrow=\left(\frac{\sigma_{1}}{\beta}\right)^{\alpha}+\left(\frac{\sigma_{2}}{\beta}\right)^{\alpha}+\left(\frac{\sigma_{3}}{\beta}\right)^{a}
$$

where $\sigma_{1}, \sigma_{2}$ and $\sigma_{3}$ are principle stresses. Equation (23) is the essence of Weibull aralysis. The issue of other possible forms for $\psi$ has been discussed in detail in articles by Duffy and Amold (1990), Duffy and Manderscheid (1990), Thomas and Wetherhold (1991), and Duffy et al. (1993).

As a final note, equations (20) and (23) can be equated once a distribution function is specified for the resistance random variable. As was indicated earlier, the distribution of choice is the Weibull distribution. There is a fundamental reason for this choice that goes beyond the fact that the Weibull distribution usually provides a good fit to the data. Often times the lognormal distribution provides an adequate fit to failure dara representing ceramic materisls. However, the log-normal distribution precludes any accounting of size effects. The reader is directed to work by $\mathrm{Hu}$ (1995) for a detailed discussion on this matter. As it turns out, once a conscious choice is made to utilize the Weibull distribution, equations (20) and (23) provides a convenient formulation for parameter entimation. The details 
for sccomplishing this are provided in Duffy (1995).

The next issue the design engineer is confronted with concerns the numerical evaluation of equation (13) when a closed form solution is not readily available. The remainder of this article is dedicated to this important issue. However, before proceeding on to the next section the reader is reminded that probsbilistic concepts were introduced by adopting a very simple failure criterion. In the sections that follow the failure criterion is left unspecified and the details of the numerical techniques are highlighted.

\section{MONTE CARLO METHODS}

In this section the authors expand the scope of the discussion beyond simple failure criterion represented by safety factors and safety margins. This discussion begins with the observation that most structural components are designed based on the results obtained from a finite element analysis. This analysis can incorporate a mechanical analysis, a thermal analysis, or both. In all cases the design engineer seeks to predict, and most tines minimize, the stress field throughout the component in an economical fashion. The stress field is approximated by the stress state obtained from each discrete element. Once again the focus of the design algorithms presented here is on individual (discrete) elements. In general the reliability of an individual finite element is computed from the expression

$$
R=\operatorname{Probabiling}\left[g\left(y_{*}\right)>0\right]
$$

when a fallure criterion is used to define point failure. Note that the failure criterion is left unspecified thus equation (25) is a general, fundamental relationship. The discussion that follows outlines specific details that must be embedded in this relationship.

To evaluate equation (25) the design space must be defined. In addition, the relevant joint probability density function that represents the design variables must be established. As was indicated earlier if there are $N$ random variables associated with a limit state function, then the design space is an $N$-dimensional space (a hyperspace) that represents the entire domain of possible values of the design variables. In order to transform a limit state function into a reliability model a joint probability density function must be utilized. This function establishes the relative frequency of occurrence for a specific combination of values (realizations) of the design random variables. Keep in mind that the limit state function is used to determine which area of the design space (a region that represents ill possible outcomes of the design random variables) will result in a successful event. Thus according to equation (25) the safe domain of the design space should satisfy $g\left(y_{-}\right)>0$. Obviously, the portion of the design space that satisfies $g\left(0_{0}\right) \leq 0$ is the failure domain for the finite element. Thus the reliability of a finite element is the integration of the joint probability density function over the safe design space defined by the failure criterion. This integration takes the form

$$
R=\int_{0} f\left(y_{s}\right) d y_{s}
$$

where $f\left(y_{-}\right)$is the joint density function of the random variables, and $\delta$, is the safe domain of the design space. This concept is simplified to a two-dimensional design variable space depicted in Figure 2.

Unfortunately, the integral in equation (26) does not usually have a closed form solution. An exception to this was presented in the section where the principles of Weibull analysis are examined. Thus in general, numerical techniques must be urilized to evaluate the reliability of a finite element. Two numerical techniques are discussed in this article that provide approximate solutions of equation (26). They are the conventional Monte Carlo method, and the fast probability integration (FPI) method. Other methods exist (the reader is referred to $\mathrm{Hu}$ (1995) for an overview) but only these two methods are presented here due to limitations placed on the length of this article. The reader is referred to Wu (1994) for a more comprehensive development of the numerical techniques associated with the FPI method. In addition, Hu's thesis (1995) presents details regarding a Monte Carlo method with an extremely efficient sampling approach.

The conventional Monte Carlo simulation is conceptually simple, very general, and relatively straightforward to implement. Thus it is commonly used to numerically estimate the probability of failure when a closed form solution to equation (26) is unavailable. In general the probability of failure of a structural component can be expressed as

$$
P_{f}=\int_{\theta_{f}} f\left(y_{s}\right) d y_{s}
$$

where $\delta_{f}$ is the failure domain that satisfies the expression

$$
g\left(y_{e}\right) \leq 0
$$

Equation (27) is an alternative expression to equation (26) since

$$
P_{\rho}=1-R
$$

Now define an indicator function $I$ such that 


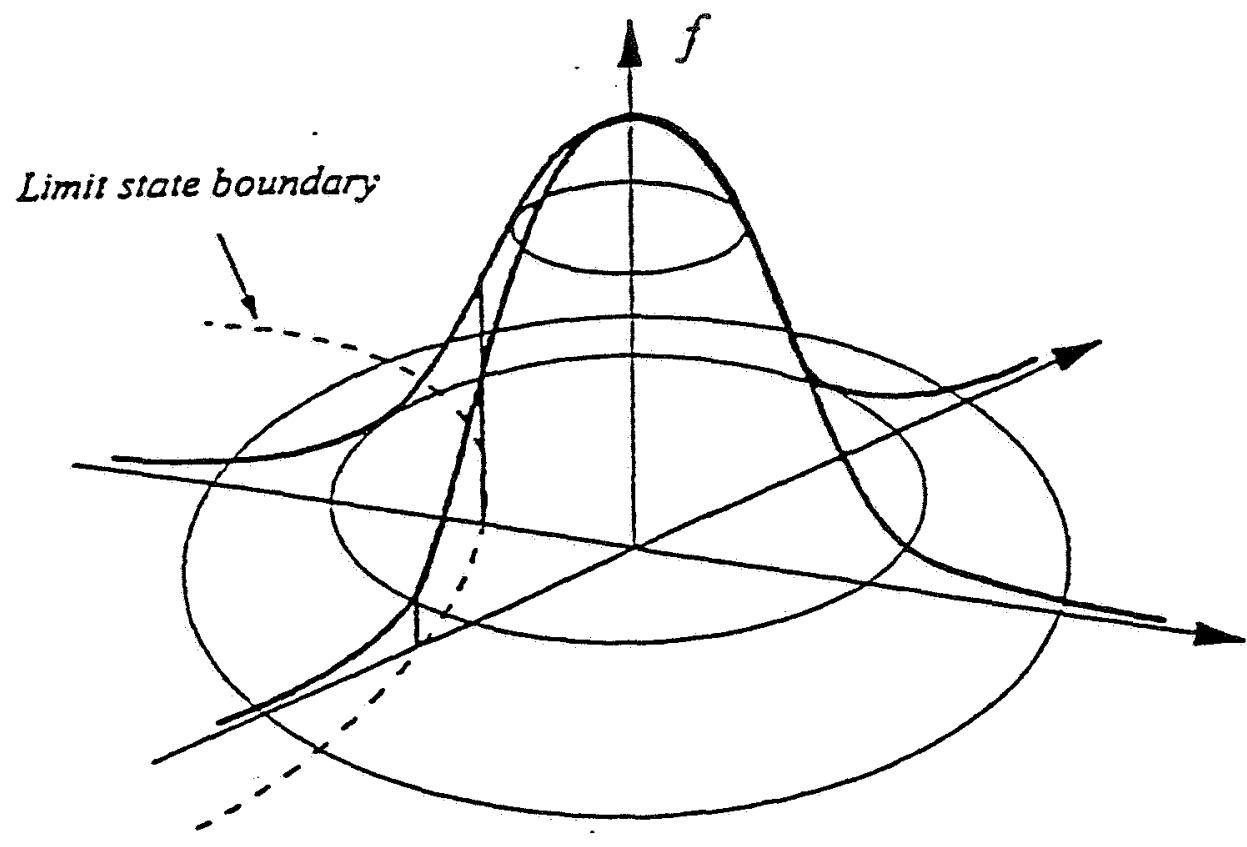

Figure 2 Two-dimensional joint PDF with limit state

$$
I= \begin{cases}1 & g\left(y_{e}\right) \leq 0 \\ 0 & g\left(y_{e}\right)>0\end{cases}
$$

This indicator function can be included in the integral defined by equation (27) if the integration range is expanded to include the range of the design space, i.e.,

$$
P_{f}=\int_{g,+b_{f}} I f\left(V_{e}\right) d y_{e}
$$

The integral on the right side of this expression defines the expectation of the indicator function, i.e.,

$$
E[\Pi]=\int_{s_{s}-b_{j}} I f\left(y_{e}\right) d y_{e}
$$

Recall from statistics that the definition of the mean $(\mu)$ of a random variable is the expectation of the variable. Thus
Also recall that the mean associated with a random varieble can be estimated from a sample taken from the population that is being chancterized by the distribution function $f(x)$. The estimated value of the mean is given by the simple expression

$$
\bar{x}=\frac{1}{N} \sum_{j=1}^{N} x_{j}
$$

Where $x_{j}$ is the th observation in 2 random ample taken from the population. In 2 similas fashion the probability of fallure $\left(P_{f}\right)$ represents the expected value, of the indicator function. Thus equation (31) can be expressed as

$$
\left.P_{f}=\lim _{N-\left\{\frac{1}{N}\right.} \sum_{j=1}^{N} I_{j}\right\}
$$

Here it is implied that a random sample of successes $(I=1)$ and failures $(I=0)$ has been generated. Thus $I$, is the the evaluntion of the limit state function where the random observections have 
been generated from the cumulative distribution function $F_{Y_{\mathrm{s}}}$.

The simulation method defined by equation (35) is referred to as conventional Monte Carlo simulation. The objective is to generate sufficiently large set of observations (i.e., large $N$ ) in order to reproduce the statistical characteristics of the underlying population that the observations are taken from. The concept of the conventional Monte Carlo method is shown in Figure 3, where the solid circles represent a success, i.e., $I=1$ and $g\left(y_{-}\right) \leq 0$. The open circles are observations that do not pass the failure criterion. Here $I=0$ and $g(f)>0$. While this approsch may not be the most efficient numerical technique, eventually it will converge to the correct solution, i.e., the solution approaches $P_{f}$ in the limit as $N$ approaches infinity.

\section{THE FAST PROBABILITY INTEGRATION METHOD}

This section presents the details of obtaining component reliabilities from fast probability integration (FPD methods. These details are presented in terms of the simplified failure criterion defined by safety factors or safety margins. This is done to merely clarify technical concepts. At the end of the section the details are provided that allows the applieation of this method to arbitrary failure criterion.

Thus the probability of failure for a structural component can be expressed as

$$
P_{f}=\operatorname{Probabiliy}(R-L \leq 0)
$$

where $R$ is the resistance random variable and $L$ is the load random variable both of which were stipulated as design variables). Define the safery margin as

$$
\boldsymbol{M}=\boldsymbol{R}-\boldsymbol{L}
$$

The expectation of the safery margin is

$$
\mu_{M}=\mu_{x}-\mu_{L}
$$

where $\mu_{M}$ is the mean of $M, \mu_{R}$ is the mean of $R$ and $\mu_{L}$ is the mean of $L$. Similarly, the variance of the safety margin is given by the expression

$$
\delta_{L}^{2}=8_{R}^{2}+8_{L}^{2}-2 \operatorname{cov}[R, L]
$$

where "cov" represents the covariance function, $\delta_{z}^{2}$ is the variance of $R$, and $\delta_{L}{ }^{2}$ is the variance of $L$. However, if $R$ and $\boldsymbol{L}$ are independent random variables, then

$$
\operatorname{cov}[R, L] \equiv 0
$$

and

$$
\delta_{L}^{2}=\delta_{R}^{2}+\delta_{L}^{2}
$$

Making use of the definition of the safety margin, the probability of failure can be expressed as

$$
P_{f}=\text { Probability }(-\infty \leq M \leq 0)
$$

If $R$ and $L$ are nomal random variables, then $M$ (which is also a linit state function) becomes a linear combination of two normally distributed random variables. Thus $M$ is a normally distributed random variable. Making use of the standard normal CDF ( $\$$ ), the probability of failure is given by the expression

$$
P_{f}=\Phi\left(-\frac{\mu_{\mu}}{\delta_{\mu}}\right)
$$

Substitution yields

$$
P_{f}=\Phi\left[\frac{\mu_{L}-\mu_{x}}{\left(\delta_{L}^{2}+\delta^{2}\right)^{12}}\right]
$$

Now define the reliability index $\beta$ such that

$$
B=\frac{\mu_{R}-\mu_{L}}{\left(\delta_{R}^{2}+\delta_{L}^{2}\right)^{1 / 2}}
$$

then

$$
P_{f}=\Phi(-\beta)
$$

This is equivalent to the integral expression given in equation (19), i.e.,

$$
P_{f}=\int_{d} f(R, L) d R d L
$$

Thus equation (46) represents a "fast integration" of equation (47), hence the origin of term "fast probability integration" (FPI) for the approach that utilizes the reliability index. The expression 


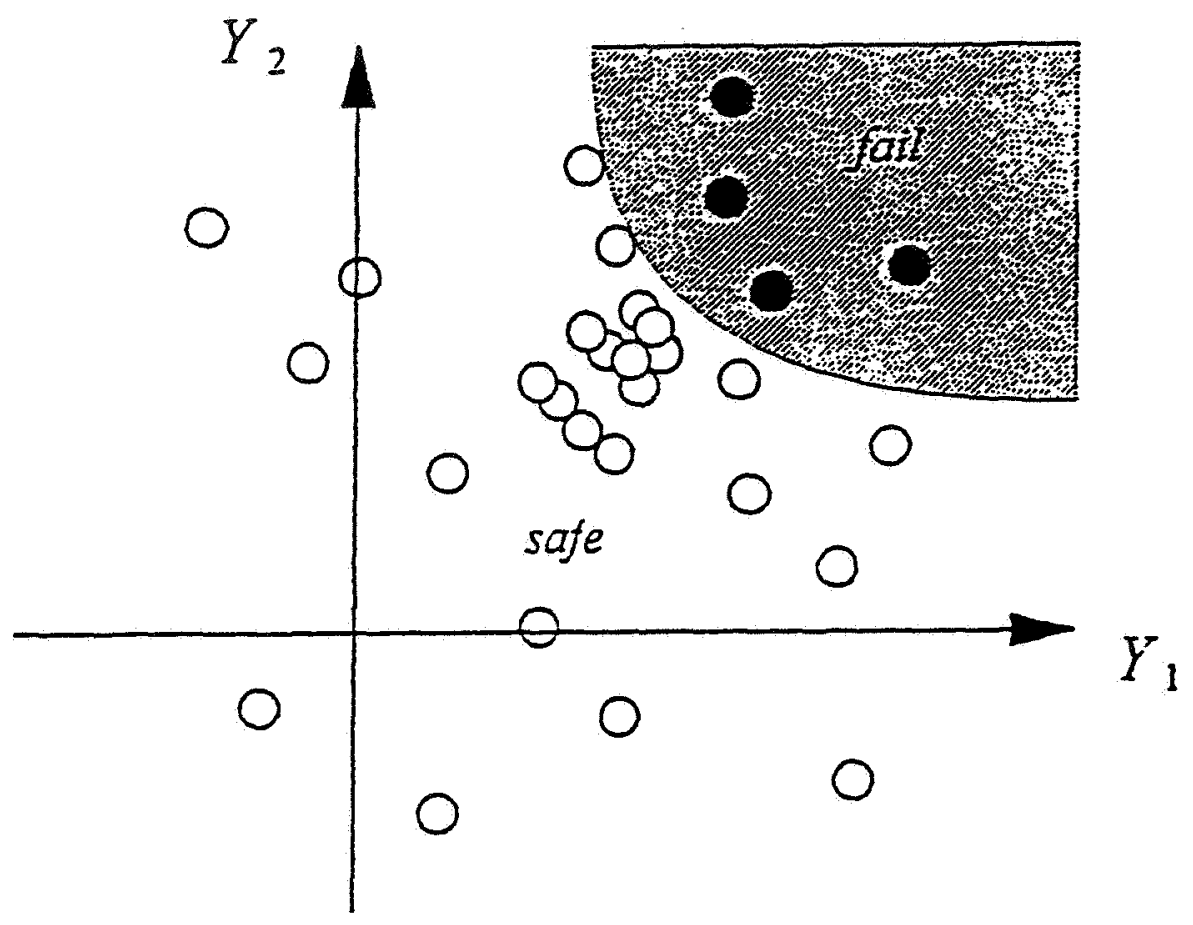

Figure 3 Conventional Monte Carlo methods

for the reliability index was derived based on the special case where the safety margin serves as the limit state function. More generally, other expressions for $\beta$ can be derived by adopting different limit state functions. However, in general, the straightforward relationship between the reliability index and the probability of failure expressed above no longer holds. If the limit state function is a non-linear function of the design variables or the design variables are not normally distributed, then equation (46) will not hold. Yet for either case there are approximations that can be utilized which yield good results, provided the limit state functions and the design variables are suitably restricted.

Before the discussion on how the relationship between the probability of failure and the reliability index can be approximated, a geometric interpretation of the reliability index is given using the simple definition of the safery margin expressed in equation (38). If $\boldsymbol{R}$ and $\boldsymbol{L}$ are normally distributed, they can be transformed to standard normal variables. By definition the transformed resistance variable is

$$
R^{\prime}=\frac{R-\mu_{x}}{\delta_{x}}
$$

and the transformed load variable is

$$
L^{\prime}=\frac{L-H_{L}}{\delta_{L}}
$$

Thus the resistance variable can be expressed as

$$
R=R^{\prime} \delta_{x}+\mu_{x}
$$

and the load variable can be expressed as

$$
L=L^{\prime} \boldsymbol{\delta}_{L}+\boldsymbol{H}_{L}
$$

In terms of the transformed random variables the safety margin becomes

$$
M=\delta_{R} R^{\prime}-\delta_{2} L^{\prime}+\left(\mu_{2}-\mu_{L}\right)
$$

Now the reliability index can be interpreted as the shortest distance from the origin in the transformed variable space to the frilure surface, which is defined by $\boldsymbol{M}$. This is depicted in Figure 4 where the failure surface associnted with thir particular safety margin is shown in both the original and the transformed design variable space. The point on the failure surface that is 


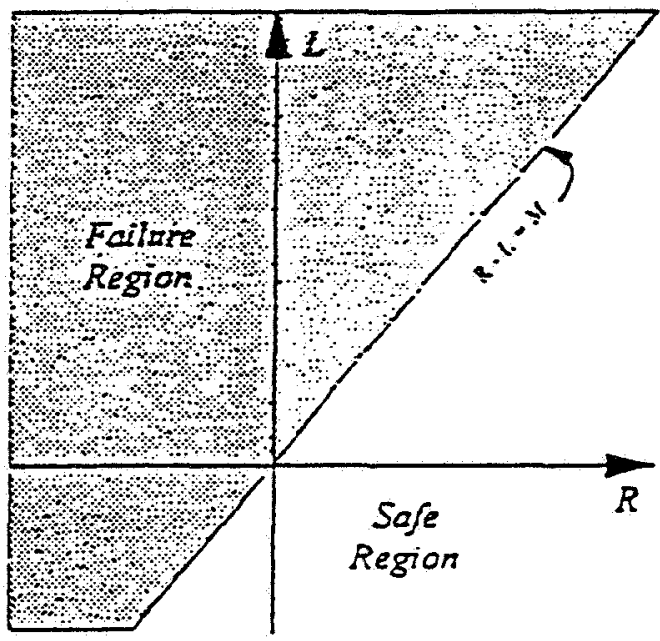

Figure 4 a) Failure surface in the standard variable space.

nearest to the origin is referred to as the most probable point (MPP) in structural reliability literature. The reader can easily verify that equation (45) can be derived from the geometry presented in Figure 4. This last figure is important. If

- the limit state function can be linearized, and

- the design variables can be transformed to standard normal variables,

then $\beta$ can be determined using anslytic geometry concepts. Once $\beta$ has been found, the probability of failure is calculated directly using equation (46).

Finally, before discussing approximate methods associated with linearizing the limit state function and normalizing the design variables, a brief diseussion is necessary concerning the extension of equation (46) to $N$ design variables. The preceding discussion focused on two independent, normally distributed, random variables. Equation (47) holds for $N$ independent, normally distributed random variables, if the limit state function $(g)$ is a linear function of the random variables. Under these circumstances

$$
B=\frac{\mu_{8}}{8_{8}}
$$

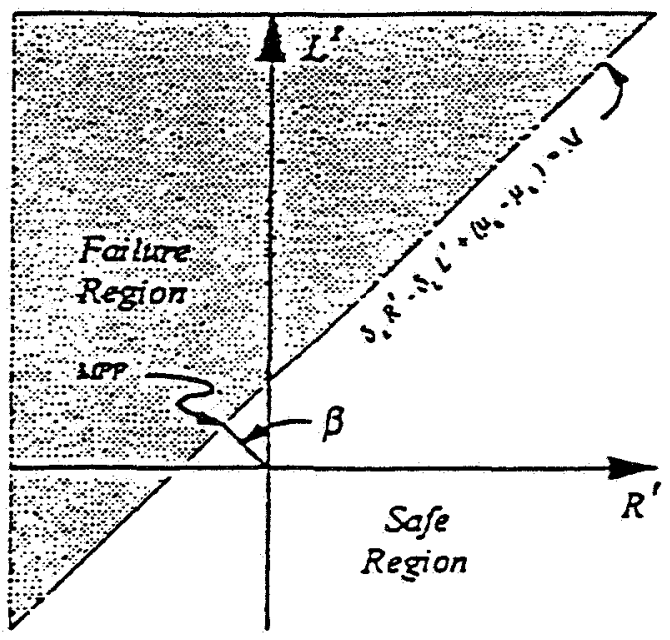

b) Failure surface and MPP in the transformed variable space

where

$$
8=1-\sum_{i=1}^{N} a_{i} y_{1}
$$

$$
H_{2}=E[g]
$$

and

$$
8^{2}=\operatorname{VAR}[g]
$$

Note that $a_{l}$ is the coefficient of the $i$ th term of the limit state function and depends on the particular limit state function utilized.

\section{THE HASOFER-LIND APPROXIMATION}

Hasofer and Lind (1974) proposed a technique (identified here as the H-L method) that approximates the failure surface for those cases where the limit state function is not a linear combination of the design variables. The failure surface (2 hyper-surface in the $N$-dimensional design variable space) is approximated by a hyper-plane tangent to the frilure surface at 
the MPP (see Figure 4). This approximation is accomplished by utilizing the first term of a Taylor series expansion of the limit state function at the MPP. Thus

$$
g\left(z_{\varepsilon}\right)=g\left(z_{i}^{*}\right)+\sum_{i=1}^{H}\left(\frac{\partial g}{\partial z_{i}}\right)_{0}\left(z_{i}-z_{i}^{*}\right)
$$

where $z$ is the vector of standard normal variables which are selated to the design variables in the following manner

$$
z_{s}=\frac{\left(y_{s}-\mu_{r_{s}}\right)}{\delta_{r_{0}}}
$$

Here $2^{\circ}$ is the vector representing the location of the MPP, and the asterisk associated with the partial derivative indicates the vector and the associated derivatives are being evaluated at the MPP.

Since the linit state function is approximated by the first term of a Taylor series expansion, the $H-L$ method is referred to as a "first order" method. Keep in mind that the H-L approximation will be exact if the design variables are nomally distributed, and the true linit state function is linear. The reader should question how good the approximation is if the actual limit state function is not linear (a hyper-plane) in the transformed standard normal variable space. The joint probability density function tends to decay exponentially with a relative increase in distance from the mean (i.e., the "peak" of the joint PDF in Figure 2). For large values of $\beta$ (i.e., low probability of failure) the main contribution to the probability integral, i.e., equation (28), usually comes from regions nesr the MPP. since the relevant functional values of the joint PDF will assume their largest values in the near vicinity of the MPP. Therefore, provided that the actual linit state surface is well-behaved and does not exhibit significant deviations from the tangent hyperplane approximation in the neighborhood of the MPP, a reasonably accurate estimate of the actual probability of failure $(P)$ can still be obtained from equation (46) by this first order approximation.

Since $\beta$ represents the shorest distance from the origin to the failure surface in standard normal variable space, and the location of the MPP is not known a priori, a search algorithm must be employed. An optinization method making use of Lagrange multipliers is utilized here. The following steps represent the details of this search algorithm:

(1) Assume initial values for the normal design variables $y_{1}$, and transform these values to standard normal values $z_{i}^{*}$ using equation (58).

(2) Transform the limit state function $\left.g \sigma_{\text {. }}\right)$ to $g\left(z_{-}\right)$using equation (57). This requires the evaluation of the

partial derivatives at the corresponding values of $z^{*}$.

(3) Evaluate the Lagrange multipliers

$$
\alpha_{i}^{*}=\left\{\frac{\left(\frac{\partial_{g}}{\partial z_{1}}\right)}{\left[\sum_{1+1}^{n}\left(\frac{\partial_{z}}{\partial z_{1}}\right)^{2}\right]^{1 / 2}}\right\}
$$

(4)

$$
g\left(z_{0}\right)=0
$$

and solve for $\beta$. Note that this last expression is a scalar valued function. Hence one equation is solved for one unknown $(\beta)$.

(6) With $\beta$ known, update values of $y^{\circ}$ and repear steps (3) to (5) until a suitable convergence criterion is met. This convergence criterion can be easily related to the change in $\beta$ from one iteration to the next.

The geometric interpretation of the algorithm above is shown in Figure 5. Note that the rate of convergence for the HL algorithm will depend on the following

- the nature of the true limit state function,

- the starting point,

- the characteristics of the random variables, and

- the correlation between the random variables.

These issues have been discussed thoroughly in the open literature and will not be revisited here.

\section{THE RACKWITZFIESSLER APPROXIMATION}

If the random variables that are utilized in the limit sate function are not normally distributed, a second approximation must be employed. Based on a concept suggested by Paloheino and Hannus (1974), Rackwitz and Fiessler (1978) proposed a modification of the FPI method to account for design variablea 


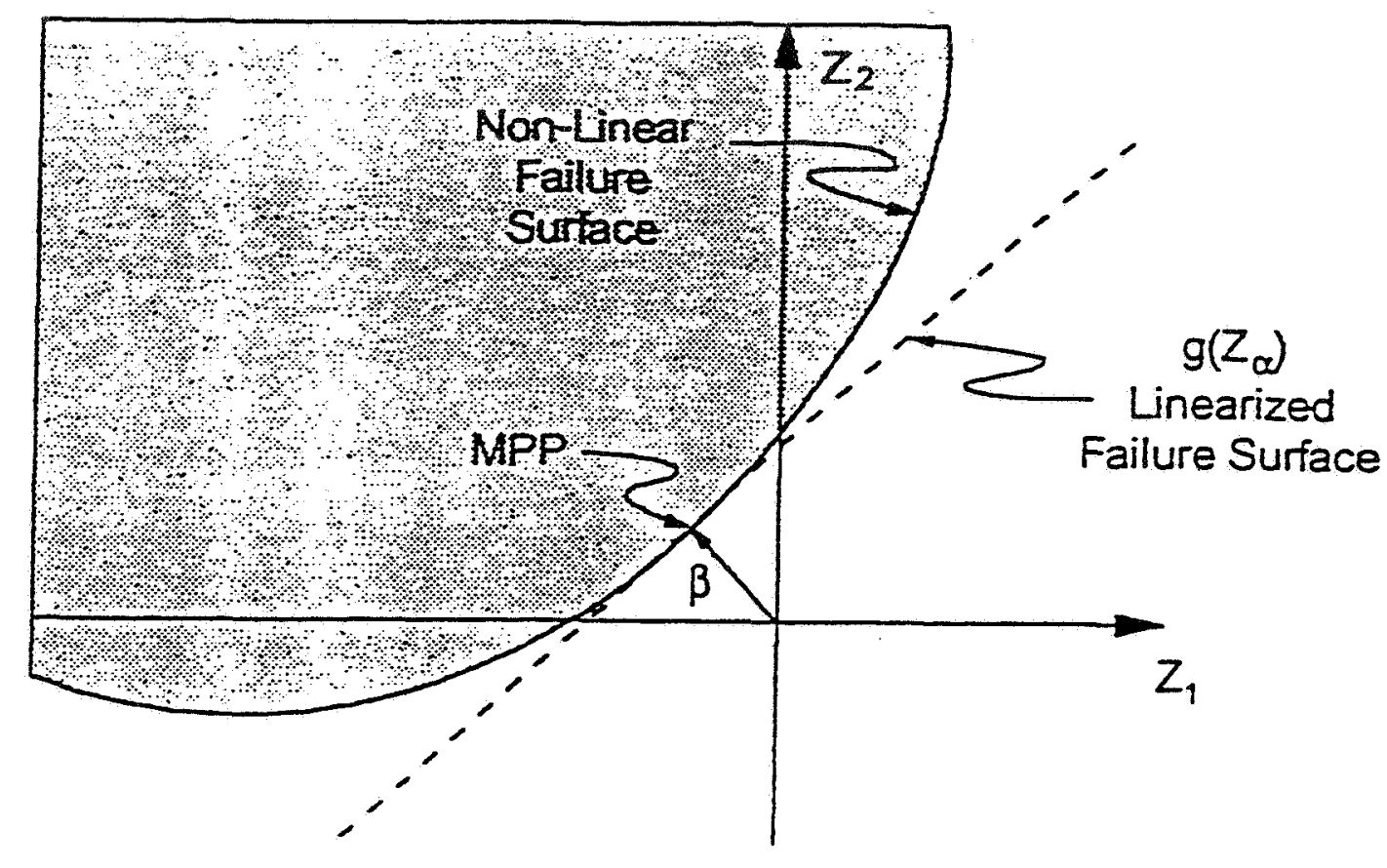

Figure 5 Schematic of the Hasofer-Lind approximation where a non-linear failure surface is approximated by a linear tangent hyper-plane

with non-normal distributions. The technique (referred to here as the R-F method) converts non-nomal random variables into standard normal variables by first equating the CDFs of the standard normal and non-normal distributions, i.e.,

$$
\Phi\left(\frac{y_{i}^{*}-\mu_{r_{1}}^{R}}{\delta_{Y_{i}}^{A}}\right)=F_{Y_{i}}\left(y_{l}\right)
$$

Here $F_{Y_{1}}$ represents the non-nomal cumulative distribution function (e.g., the two-parameter Weibull distribution) and $\Phi$ is the standard nomal cumulative distribution function. In addition the PDFs of the standard normal and non-normal distributions are equated leading to the expression

$$
\frac{1}{\delta_{r_{1}}^{N}} \phi\left(\frac{y_{i}^{*}-\mu_{r_{1}}^{A}}{\delta_{r_{1}^{N}}^{N}}\right)=f_{r_{1}}\left(\theta_{1}\right)
$$

Here $f_{Y_{1}}$ represents the non-normal probability density function and $\$$ is the standand normal probability density function. These last two expressions must be evaluated at every approximated MPP. The character $\vec{N}$ signifies the normal distribution in both expressions. Thus, the equivalent nomal mean $\left(\mu_{r_{i}}{ }^{\phi}\right)$ and equivalent normal standard deviation $\left(\delta_{Y_{1}}^{A}\right)$ of non-normal variables can be derived from equations (62) and (63). Specifically

$$
\mu_{x_{i}}^{A}=y_{i}^{*}-\delta_{y_{i}}^{A} \phi^{-1}\left[F_{Y_{1}}\left(y_{i}\right)\right]
$$

and

$$
\delta_{Y_{1}}^{A}=\frac{\phi\left[\Phi^{-1}\left[F_{Y_{1}}\left(y_{1}\right)\right]\right]}{f_{Y_{1}}\left(y_{1}\right)}
$$

\section{SUMMARY}

An overview is given of engineering concepts and computational algorithms which have been developed enabling probabilistic design approaches to structural analysis. Probabilistic design approaches are shown to have evolved as a natural extension of traditional deterministic design spprosches.

The well established Weibull analysis approach, commonly utilized for the design of components fabricated of 
brittle materials such as ceramics, is shown to be a special case of the more general probabilistic design problem formulation. Substantial developments have occurred to extend the Weibull analysis approsch to more complex failure mechanisms and for the approximate numerical solution of more realistic component design problems using finite element analysis techriques. These efforts have produced comprehenaive design tools, such as is embodied in the CARES family of sottware developed at NASA Lewis Research Center.

In the more general case, much of the development effort has focused on efficient numerical algorithms to achieve accurate approximate solutions of probabilistic design problems involving complex and nonlinear failure or limit state functions and design parameters described by non-normal distributions. Several specific algorithms are described which are extensions of the fast probability integration approaches originally developed by Hasofer and Lind, and Rackwitz and Feissler, as well as extensions of Monte Carlo simulation approaches allowing for more selective sampling. Again, these efforts have produced comprehensive design tools, such as is embodied in the NESSUS family of software developed at Southwest Research Institute under the sponsorship of NASA Lewis Research Center.

The various methods presented provide a quantitative basis to account for design uncertainties inherent to physical systems. The ultimate benefit of probabilistic design approaches is a more rational basis for maling design decisions that balance component or system efficiency with reliability or safery. This benefit is especially important in the design of high-performance and/or life-critical systems.

\section{REFERENCES}

Drucker, D.C., Greenberg, W. and Greenberg, H.J., "Extended Limit Design Theorems for Continuous Media," Quart. Appl Math., Vol. 9, pp. 381-389, 1952.

Duffy, S.F. and Arnold, S.M., "Noninteractive Macroscopic Statistical Failure Theory for Whisker Reinforced Ceramic Composites," Joumal of Composite Materials, Vol. 24, No. 3, pp. 293-308, 1990.

Duffy, S.F. and Manderscheid, J.M., "Noninteractive Macroscopic Reliability Model for Ceramic Matrix Composites with Orthotropic Material Symmetry," Journal of Engine ering for Gas Turbines and Power, Vol. 112, No. 4, pp. 507-511, 1990.

Duffy, S.F., Chulya, A. and Gyekenyesi, I.P., "Structural Design Methodologies for Ceramic Based Material Systems," Chapter 14 in Flight-Vehicle Materials, Structures and Dynamics Technologies - Assessment and Future Directions, eds. A.K. Noor and S.L. Venneri, The Ameriean Society of Mechanical Engineers, pp. 265-285, 1992.
Duffy, S.F., Palko, J.L. and Gyekenyesi, J.P., "Structural Reliability Aralysis of Laminated CMC Components, Journal of Engineering for Gas Turbines and Power, Vol. 115, No. 1, gp. 103-108, 1993.

Duffy, S.F., "Issues Relating to Reliability Based Design Component Design," to sppear in Handbook on Whisker and Particulate Reinforced Ceramic Matrix Composites, K Bowman, S.K. El-Rahaiby, and J. Wachtman eds., to be published by DoD Ceramics Information Analysis Center.

Hasofer, A.M., and Lind, N.C., "An Exact and Invariant First Order Reliability Format," Joumal of the Engineering Mechanies Division, Vol. 100, No. EM1, pp. 111-121, 1974.

Hu, J., "Modeling Size Effects and Numerical Techniques in Structural Reliability Analysis," Masters Thesis, Cleveland State University, 1995.

Paloheimo, E. and Hannus, M., "Structural Design Based on Weighted Fractiles," Joumal of the Structural Division, Vol. 100, No. ST7, pp. 661-676, 1974.

Rackwitz, R. and Fiessler, B., "Structural Reliability Under Combined Random Load Sequences," Journal of Composise Structures, Vol. 9, pp. 489-494, 1978.

Thomas, D.J. and Wetherhold, R.C., "Reliability Analysis of Laminates with Load Sharing," Joumal of Composize Materiats, Vol. 25, pp. 1459-1475, 1991.

Weibull, W., "A Statistical Theory of the Strength of Materials,"Ingeniors Vetenskaps Akademien Handlinger, No. 151, 1939.

Wu, Y.-T., "Computational Methods for Efficient Structural Reliability and Reliability Sensitivity Aralysis," AlA Joumal, Vol. 32, No. 8, pp. 1717-1723, 1994. 